\title{
$M$-Band Compactly Supported Orthogonal Symmetric Interpolating Scaling Functions
}

\author{
Peng-Lang Shui, Zheng Bao, Senior Member, IEEE, and Xian-Da Zhang, Senior Member, IEEE
}

\begin{abstract}
In many applications, wavelets are usually expected to have the following properties: compact support, orthogonality, linear-phase, regularity, and interpolation. To construct such wavelets, it is crucial designing scaling functions with the above properties. In two- and three-band cases, except for the Haar functions, there exists no scaling function with the above five properties. In $M$-band case ( $M \geqslant 4)$, more free degrees available in design enable us to construct such scaling functions. In this paper, a novel approach to designing such scaling functions is proposed. First, we extend the two-band Dubuc filters to $M$-band case. Next, the $M$-band FIR regular symmetric interpolating scaling filters are parameterized, and then, $M$-band FIR regular orthogonal symmetric interpolating scaling filters (OSISFs) are designed via optimal selection of parameters. Finally, two family of four-band and five-band OSISFs and scaling functions are developed, and their smoothnesses are estimated.
\end{abstract}

Index Terms-Cardinal interpolation, linear-phase, scaling function, Sobolev exponent, wavelet sampling.

\section{INTRODUCTION}

A COMPACTLY supported wavelet is typically constructed from a compactly supported scaling function that generates a multiresolution analysis. It is important (and nontrivial) to construct scaling functions (and hence wavelets) with desirable properties, such as orthogonality, high regularity, symmetry/linear phase, and interpolation. However, in the two-band and three-band cases, no scaling function can provide all the above properties. Daubechies [1] had shown that, except for the Haar function, there exists no two-band compactly supported orthogonal symmetric scaling function. Nevertheless, the $M$-band $(M>2)$ scaling functions or wavelets have appeared that partake several of the above five properties; for examples, see [2]-[9]. The multiscaling functions and multiwavelets with compact support, orthogonality, continuity, and cardinal interpolation were designed [10]. These owe to more free degrees available in design. In fact, when $M \geqslant 4$, one can design the $M$-band ( $M \geqslant 4$ ) scaling functions as much as wavelets with the above five advantages. In [11], Ji and Shen proposed an approach to constructing such $M$-band scaling functions from $(M-1)$-band scaling functions, and several four-band examples were presented.

As is well-known, compact support, linear-phase, regularity, orthogonality, and interpolation are important in both theory

Manuscript received May 17, 2000; revised April 4, 2001. This work was supported by the National Science Foundation of China under Grant 69831040. The associate editor coordinating the review of this paper and approving it for publication was Dr. Xiang-Gen Xia.

The authors are with Key Laboratory for Radar Signal Processing, Xidian University, Xi'an, China (e-mail: plshui@ @rsp.xidian.edu.cn).

Publisher Item Identifier S 1053-587X(01)05845-7. and applications of multirate filterbanks and wavelets. Since traditional wavelets or filterbanks do not possess all the above five advantages, in special applications, a tradeoff among required advantages has to be made. For example, in image processing, the linear phase avoids phase distortion in reconstruction, compact support provides efficient computation, and the orthonormality brings the convenience in performance analysis, but two-band wavelets or filterbanks hardly satisfy these demands. Therefore, the design of scaling functions and wavelets with the above five advantages has important practical meaning. The paper will be devoted to the design of such scaling functions via a novel approach in which we utilize the parameter representations of scaling filters with cardinal interpolation and symmetry based on $M$-band Dubuc filters.

The interpolation dates back to Shannon's sampling theorem, and from the multiresolution analysis point of view, the classical Shannon sampling uses the Sinc function as the scaling function for a bandlimited signal that provides an exact representation by its uniform samples with a sampling rate higher than its Nyquist rate. Recently, for scale-limited signals, this classical theorem has been extended to many other forms [10], [12]-[17], and these new forms are the direct avenue in the performance of the fast and accurate initialization of the wavelet series transforms (WSTs). In the two-band interpolating wavelets and subdivision scheme, the Dubuc filters play an important role [16]-[21]. Similarly, these types of filters can be extended to the $M$-band case. By these filters, the $M$-band regular scaling filters with cardinal interpolation can be parameterized, which is the cornerstone of the work in this paper.

The paper is organized as follows. In Section II, we briefly review some basic properties and structures of $M$-band scaling functions. In Section III, the $M$-band Dubuc filters are introduced, and the parametric representation of the subfilters of orthogonal symmetric interpolating scaling functions (OSISFs) is developed. In Section IV, by using parametric representation, we propose an efficient method for designing OSISFs. In Section $\mathrm{V}$, two families of scaling functions with the above five advantages are developed, and their Hölder exponents and Sobolev exponents are estimated. Finally, this paper is concluded in Section VI.

\section{OVERVIEW OF $M$-BAND SCALING FILTERS/FunCtions}

Similar to the two-band case, one usually uses a multiresolution analysis (MRA) with a scaling factor of $M$ to construct $M$-band wavelets. One first constructs a scaling filter and function and then the wavelet filters and wavelets. Once a scaling filter or scaling function is available, one can design $M$-band wavelet filters and wavelet bases via different approaches (such 
as the state-space approach and the factorization approach) [4]-[6]. $M$-band wavelets or filterbanks have more advantages than two-band wavelets. Generally speaking, the wavelet filters and wavelet bases can keep some good properties of the scaling functions such as the linear-phase and smoothness. Therefore, in order to obtain a wavelet system, the linchpin is to design a scaling function with good properties. In what follows, we briefly review the fundamental results on $M$-band scaling filters and functions.

A real orthogonal scaling filter is a sequence $h_{0}(n)$ that satisfies the following linear and quadratic constraints:

$$
\sum_{k} h_{0}(k) h_{0}(k+M l)=\delta(l), \quad \sum_{k} h_{0}(k)=\sqrt{M}
$$

Let $£(M)=\{\ldots,-2 M,-M, 0, M, 2 M, \ldots\}$ and $\Re(M)=$ $\{0,1,2, \ldots, M-1\}$, where $£(M)$ is a lattice generated by $M$, and $\Re(M)$ is the set of representatives of $£(M)$. In this way, the scaling filter $h_{0}(n)$ can be represented by its polyphase components or subfilters, i.e.,

$$
\begin{aligned}
H_{0}(z) & =\sum_{n} h_{0}(n) z^{-n}=\sum_{i \in \Re(M)} \sum_{n} h_{0, i}(n) z^{-M n-i} \\
& =\sum_{i \in \Re(M)} z^{-i} H_{0, i}\left(z^{M}\right)
\end{aligned}
$$

where $h_{0, i}(n)=h_{0}(M n+i)$ are referred to as the subfilters, whereas $H_{0, i}(z)$ is the polyphase component. It is easy to show that a real scaling filter $h_{0}(n)$ is orthogonal iff

$$
\begin{aligned}
\sum_{i \in \Re(M)} H_{0, i}(z) H_{0, i}\left(z^{-1}\right) & =1 \\
\text { or } \frac{1}{M} \sum_{i \in \Re(M)} H_{0}\left(\frac{\omega-2 \pi i}{M}\right) H_{0}^{*}\left(\frac{\omega-2 \pi i}{M}\right) & =1 .
\end{aligned}
$$

Given a scaling filter, the associated scaling function is the solution of the following two-scale difference equation:

$$
\psi_{0}(x)=\sqrt{M} \sum_{n} h_{0}(n) \psi_{0}(M x-n)
$$

However, an orthogonal scaling filter does not always derive an orthogonal scaling function. A necessary condition for (4) to be a solution in $L^{1}(\mathbf{R})$ is that the scaling filter satisfies the linear constraint $\sum_{n} h_{0}(n)=\sqrt{M}$. Moreover, when $h_{0}(n)$ is a finite impulse response (FIR), via the infinite product of matrices and the joint spectrum radius, the sufficient condition for (4) to exist as a unique solution in $L^{1}(\mathbf{R}) \cap L^{2}(\mathbf{R})$ is given [22]-[24]. When an $M$-band orthogonal scaling filter satisfies $H_{0}(\omega) \neq$ $0, \quad \forall \omega \in[-(\pi / M),(\pi / M)]$, the associated scaling function generates an orthonormal basis [6].

For a scaling filter, the regular order is closely related to the smoothness and the approximation power for smoothing signals of the associated scaling function, and its smoothness dictates the smoothness of the derived wavelet system. Thus, a proper regular order is required for design. An $M$-band scaling filter is said to be $K$-regular iff $H_{0}(z)$ has a polynomial factor of the form $P^{K}(z)$, with $P(z)=(1 / M) \sum_{i \in \Re(M)} z^{-i}$ for maximal possible $K$. That is

$$
H_{0}(z)=P^{K}(z) Q(z)
$$

$K$-regularity is equivalent to $H_{0}\left(e^{j 2 \pi i / M}\right)=\sqrt{M} \delta(i)$, and its derivatives from the first to the $(K-1)$ order vanish at $z=e^{j 2 \pi i / M}, i \in \Re(M)$. The smoothness of scaling functions is an important index in designing scaling functions, and the quantities to be used measure smoothness are the ones used in [11] and [26]-[28]. Define

$$
\kappa_{p}=\sup \left\{\kappa: \int_{\mathbf{R}}\left(1+|\omega|^{p}\right) \kappa\left|\hat{\psi}_{0}(\omega)\right|^{p} d \omega<\infty\right\} .
$$

When $p=2$, finiteness of the above integral defines the function $\psi_{0}(x)$ to be in Sobolev space $\mathbf{W}_{2} \kappa(\mathbf{R})$, and the critical exponent $\kappa_{2}$ is taken as a measure of the $L^{2}$ smoothness of $\psi_{0}(x)$, which is called the Sobolev exponent. The Höilder exponent $\kappa_{\infty}$ refers to the $L_{\infty}(\mathbf{R})$ case. By the Fourier analysis method, one easily estimates a low bound of the Höilder exponent $k_{\infty}$ with the following formula [1], [6]:

$$
\begin{aligned}
q_{j} & =\max _{\omega \in \mathbf{R}}\left|\prod_{l=0}^{j-1} Q\left(e^{-i \omega / M^{l}}\right)\right| \\
\kappa_{\infty} & \geqslant K-1 / 2-\inf _{j}\left\{\frac{\log q_{j}}{j \log M}\right\}-\varepsilon
\end{aligned}
$$

where $\varepsilon$ is an arbitrarily small positive number. The Sobolev exponents can be estimated by using the following method [11]. At first, for an integer $k$, let

$$
V_{k}=\left\{v \in l_{0}(Z): \sum_{\alpha \in Z} p(\alpha) v(\alpha)=0, \quad \forall p \in \Pi_{k}\right\}
$$

where $\Pi_{k}$ denotes the polynomials of degree $k$. Let an $M$-band scaling filter $h_{0}(n)$ with the support set $\{-N,-N+1, \ldots, N\}$ be $K$-regular, let $M a_{0}(n)$ be the autocorrelation sequence of $h_{\mathrm{O}}(n)$, and let

$$
\mathbf{H} \equiv\left[a_{0}(M k-l)\right]_{k, l \in[-2 N, 2 N]} .
$$

Then, when $\psi_{0}$ is stable, the Sobolev exponent $\kappa_{2}$ satisfies

$$
\kappa_{2}=-\frac{1}{2} \log _{M} \lambda_{a u}
$$

where $\lambda_{a u}$ is the spectrum radius of $\left.\mathbf{H}\right|_{v_{2 K-1}}$.

Define

$$
\begin{aligned}
\mu(k) & =\int x^{k} \psi_{0}(t) d t \\
m(k) & =\sum_{n} n^{k} h_{0}(n) \\
\eta(k, i) & =\sum_{n}(M n+i)^{k} h_{0}(M n+i) \\
& =\sum_{n}(M n+i)^{k} h_{0, i}(n), \quad i \in \Re(M) \\
\gamma(k, i) & =\sum_{n} n^{k} h_{0, i}(n)
\end{aligned}
$$


where

$\mu(k)$ and $m(k)$ moments of $\psi_{0}$ and $h_{0}$, respectively;

$\eta(k, i) \quad$ partial moment of $h_{0}$;

$\gamma(k, i) \quad$ moment of the $i$ th subfilter.

By [6], a scaling filter is $K$-regular iff one of the following two arbitrary conditions holds:

i) The frequency response of the scaling filter has a zero of order $K$ at the $M$ th roots of unity.

ii) For $k=0,1, \ldots, K$, the partial moments $\eta(k, i)$ is a constant independent of $i$ and only dependent on $k$.

\section{III. $M$-BAND SyMmetric INTERPOLATING SCALING FILTERS/FUNCTIONS}

The Shannon sampling theorem is one of the cornerstones in signal processing and communication theory, but it only is available for bandlimited signals. Its general forms in multiresolution spaces have been discussed extensively [8], [9], [12]-[15]. As pointed out by Xia and Zhang [12], an orthogonal scaling function must be a cardinal that supports a sampling theorem identical to the Shannon sampling theorem in form. A function $\psi_{0}(x)$ is said to be a cardinal function if $\psi_{0}(n)=\delta(n)$. Unfortunately, in the two-band case, no compactly supported orthogonal cardinal scaling function exists. By relaxing some demands, two families of the scaling functions with cardinal interpolation are developed [12], [16], [17]. A family is the orthogonal cardinal scaling functions with fast decay, and another family is the compactly supported biorthogonal cardinal ones. Fortunately, $M$-band wavelets or multiwavelets can possess the following three properties.

1) orthogonality;

2) compact support;

3) interpolation.

Two examples are three-band compactly supported orthogonal interpolating scaling functions [2], [8] and multiwavelets with cardinal interpolation [10]. In [11], several four-band examples with the above properties have been given. In this section, we will investigate how to design $M$-band compactly supported orthogonal symmetric scaling functions with cardinal interpolation.

From the wavelet sampling theorem, when $\psi_{0}(x)$ is an $M$-band cardinal interpolating scaling function and the associated scaling (or multiresolution) subspaces are defined as $\mathbf{V}_{j}\left(\psi_{0}\right) \equiv \operatorname{span}_{n}\left\{M^{j / 2} \psi_{0}\left(M^{j} x-n\right)\right\}$, then we have the wavelet sampling theorem identical to the Shannon sampling theorem of the form

$$
\begin{gathered}
f(x)=\sum_{n} M^{-j / 2} f\left(\frac{n}{M^{j}}\right) M^{j / 2} \psi_{0}\left(M^{j} x-n\right) \\
\forall f(x) \in V_{j}\left(\psi_{0}\right) .
\end{gathered}
$$

According to the above sampling theorem, the uniform samples of a signal can directly substitute for the initial approximation coefficients in the associated WST initialization. The right-hand side of (8) often provides a better approximation of $f(x)$ than one without the interpolation property, even though $f(x) \notin \mathbf{V}_{j}$.
In order to design such scaling functions, we analyze the characteristics of the scaling filters.

\section{A. Characterizations of FIR Orthogonal Symmetric Scaling Filters with Cardinal Interpolation}

Let

$$
\begin{aligned}
H_{0}(z) & =\sum_{n} h_{0}(n) z^{-n}=\sum_{i=0}^{M-1} \sum_{n} h_{0, i}(n) z^{-M n-i} \\
& =\sum_{i=0}^{M-1} z^{-i} H_{0, i}\left(z^{M}\right)
\end{aligned}
$$

be the type-I polyphase representation of the scaling filter $h_{0}(n)$, where $H_{0, i}(z)=\sum_{n} h_{0, i}(n) z^{-n}$. Then, a scaling filter can be completely described by its polyphase components $H_{0, i}(z)$ or subfilters $h_{0, i}(n)$.

Theorem 1: An $M$-band real scaling filter $h_{0}(n)$ is an orthogonal symmetric one with the cardinal interpolation property if and only if the following three conditions are satisfied:

i) $h_{0,0}(n)=1 / \sqrt{M} \delta(n)$ or $H_{0,0}(z)=1 / \sqrt{M}$;

ii) $h_{0, i}(n)=h_{0, M-i}(1-n)$ or $H_{0, i}(z)=$ $z H_{0, M-i}\left(z^{-1}\right), i=1,2, \ldots, M-1$;

iii) $2 \sum_{i=1}^{N} H_{0, i}(\omega) H_{0, i}^{*}(\omega)=1-(1 / M)$, when $M=$ $2 N+12 \sum_{i=1}^{N-1} H_{0, i}(\omega) H_{0, i}^{*}(\omega)+H_{0, N}(\omega) H_{0, N}^{*}$ $(\omega)=1-(1 / M)$, when $M=2 N$.

Proof: From the cardinal interpolation property, we easily obtain condition i). When the filter $h_{0}(n)$ is symmetric about $n=0$, we have

$$
\begin{aligned}
h_{0, i}(n) & =h_{0}(M n+i)=h_{0}(-M n-i) \\
& =h_{0}(M(1-n)+(M-i))=h_{0, M-i}(1-n)
\end{aligned}
$$

which is simply condition ii).

From i) and ii), we have $H_{0,0}(\omega) H_{0,0}^{*}(\omega)=1 / M$ and $H_{0, i}(\omega) H_{0, i}^{*}(\omega)=H_{0, M-i}(\omega) H_{0, M-i}^{*}(\omega)$, $i=1,2, \ldots, M-1$. Considering the orthogonal condition (3), we know that the condition iii) holds. Contrarily, if the above three conditions are satisfied, then the associated scaling filter is an orthogonal symmetric filter with cardinal interpolation.

Corollary 2: A 3-band FIR real scaling filter is an orthogonal symmetric scaling filter with cardinal interpolation property if and only if it is a Haar-type scaling filter.

Proof: According to Theorem 1, if a 3-band FIR real scaling filter is an OSISF, then its polyphase components must satisfy

$$
H_{0,1}(\omega) H_{0,1}^{*}(\omega)=\frac{1}{3} .
$$

Since $h_{0}(n)$ is FIR, $H_{0,1}(\omega) H_{0,1}^{*}(\omega)$ is a cosine polynomial. This implies that its solutions must have the form as $H_{0,1}(\omega)=$ $(1 / \sqrt{3}) \exp (-j m \omega)$ for some integer $m$, and hence, $h_{0,1}(n)=$ $(1 / \sqrt{3}) \delta(n-m)$, and $h_{0}(n)$ is a Haar-type scaling filter.

It is known from Corollary 2 that in order to design the regular orthogonal scaling filters with the above properties, one cannot help but consider the case of $M \geqslant 4$. 
Proposition 3: A real FIR orthogonal symmetric interpolating scaling filter $h_{0}(n)$ is $K$-regular iff its subfilters satisfy

$$
\begin{array}{r}
\gamma(k, i)=\sum_{n} n^{k} h_{0, i}(n)=\frac{1}{\sqrt{M}}\left(-\frac{i}{M}\right)^{k} \\
k=0,1,2, \ldots, K-1, i \in \Re(M)
\end{array}
$$

but for $k=K$, at least one equation in (9) does not hold.

From (7) and the results in [6], $h_{0}(n)$ is $K$-regular iff for $k=0,1, \ldots, K-1$

$$
\eta(k, i)=\sum_{n}(M n+i)^{k} h_{0, i}(n)
$$

is a constant independent of $i$. According to the cardinal interpolation, we have $\eta(k, 0)=(1 / \sqrt{M}) \delta(k)$, and thus

$$
\eta(k, i)=\frac{1}{\sqrt{M}} \delta(k) .
$$

Moreover, $\eta(k, i)=\sum_{n}(M n+i)^{k} h_{0, i}(n)=\sum_{l=0}^{k}$ $\left(\begin{array}{c}k \\ l\end{array}\right) M^{l} i^{k-l} \gamma(l, i)$. By induction, it is easy to prove that the conditions in (9) hold.

\section{B. M-Band Dubuc Filters and Parameterization of the Subfilters}

Dubuc filters [19] play a key role in the subdivision scheme, Largrange halfband filters, and two-band interpolating wavelets. By the spectrum factorization of the Largrange halfband filters, the famous Daubechies wavelets were obtained [18]. Using Dubuc filters as well as the parametric representations of general interpolating filters, Donoho wavelets [16], lifting Donoho wavelets [17], and nearly orthogonal interpolating wavelets [21] have been consecutively developed. From the interpolation theory point of view, Dubuc filters originate from algebra polynomial interpolator. For example, taking $n=0$ as the interpolating point and $\{2 n+1, n=m, m+1, \ldots, m+K-1\} \subseteq 2 \mathbf{Z}+1$ as the stencil, using the values of a signal at the stencil and $K-1$ degree polynomial, one can estimate its value at $n=0$, which equals to a linear combination of its values at the stencil. The linear combination weight vector is referred to as a $K$-order Dubuc filter, where the interpolator is derived from that which is accurate for all polynomials less than $K$ degree, and the filter's coefficients can be calculated using the Largrange interpolation formula

$$
\begin{aligned}
h_{D}^{(K, m)}(n)= & \prod_{\substack{l=m, l \neq n \\
n=m, m+1, \ldots, m+K-1}}^{m+K-1} \frac{2 l+1}{2(l-n)}
\end{aligned}
$$

Mimicking the two-band case, we can define the $M$-band Dubuc filters and Largrange $M$ th-band lowpass filters. Taking $n=0$ as the interpolating points and $\{M n+i, n=m, m+$ $1, \ldots, m+K-1\} \subseteq M \mathbf{Z}+i$ as the stencil, the interpolator derived from a $K-1$ degree polynomial can implemented by the following filter:

$$
\begin{array}{r}
h_{D, M, i}^{(K, m)}(n)=\prod_{\substack{l=m, l \neq n \\
n=m, m+1, \ldots, m+K-1 .}}^{m+K-1} \frac{M l+i}{M(l-n)}, \quad i \in \Re(M) \\
\text { n }
\end{array}
$$

We call it an $M$-band $K$-order Dubuc filter. Following the polynomial interpolation theory and (11), the $M$-band $K$-order Dubuc filters have the following characteristics.

i) The interpolators derived from $h_{D, M, i}^{(K, m)}(n)$ are accurate for all polynomials whose degree is less than $K$.

ii) The filters are the solutions of the system of linear equations

$$
\begin{aligned}
& \sum_{n} n^{p} h_{D, M, i}^{(K, m)}(n) \\
& \quad=\left(-\frac{i}{M}\right)^{p}, \quad \text { for } p=0,1, \ldots, K-1 .
\end{aligned}
$$

iii) For $M=2 N, i=N$, the following relationships hold:

$$
\begin{aligned}
h_{D, 2 N, N}^{(K,-(m+K))}(-(n+1)) & =h_{D, 2 N, N}^{(K, m)}(n) \\
h_{D, 2 N, N}^{(2 K,-K)}(-(n+1)) & =h_{D, 2 N, N}^{(2 K,-K)}(n)
\end{aligned}
$$

namely, the Dubuc filter with $m=-K$ is symmetric.

iv) All coefficients of the filter are rational numbers in the form of $l / M^{K-1}$.

$M$-band Dubuc filters $h_{D, M, i}^{(K, m+k)}, k=0,1, \ldots, L-K$ just form a system of basis solutions of (12); therefore, an arbitrary $M$-band $K$-order subfilter $h_{0, i}(n)$ with support set $\{m, m+$ $1, \ldots, m+L-1\}, L \geqslant K$ can be represented as the linear combination of Dubuc filters

$$
\begin{aligned}
h_{0, i}(n)= & \frac{1}{\sqrt{M}} \sum_{k=0}^{L-K} \boldsymbol{\alpha}_{i}(k) h_{D, M, i}^{(K, m+k)}(n) \\
& \sum_{k=0}^{L-K} \boldsymbol{\alpha}_{i}(k)=1 .
\end{aligned}
$$

In the above representation, all Dubuc filters have the same order $K$, and their support sets slide on $\{m, m+1, \ldots, m+$ $L-1\}$ from the left to the right. Moreover, one easily derives the other parametric representation of the $M$-band $K$-order subfilter $h_{0, i}(n)$, that is, which is represented as the linear combination of different-order Dubuc filters. The proper parametric forms often bring some benefit in numerical computation. The another parametric representation will be discussed in the next section.

\section{Largrange Mth-Band Filters and M-Band Daubechies Wavelets}

In [18] and [25], the relationship between the Largrange halfband interpolating filters and Daubechies filters is revealed, which shows that the commonly used Largrange interpolating filters are in one-to-one correspondence with the convolutional 
squares of Daubechies filters for orthonormal wavelets of compact support. That is

$$
\begin{aligned}
H_{2,2 K}^{L \arg \text { range }}(z) & =1+\sum_{n=-K}^{K-1} h_{D, 2,1}^{(2 K,-K)}(n) z^{-(2 n+1)} \\
& =H_{2, K}^{\text {Daubechies }}(z) H_{2, K}^{\text {Daubechies }}\left(z^{-1}\right)
\end{aligned}
$$

where $H_{2,2 K}^{L \arg \text { range }}(z)$ and $H_{2, K}^{\text {Daubechies }}(z)$ denote $2 K$-regular Largrange halfband filter with length $4 K-1$ and $K$-regular Daubechies filter with length $2 K$, respectively. This relationship provides a convenient avenue to generate and analyze the Daubechies wavelets. Using the method similar to [1], $M$-band orthonormal wavelets are constructed in [6] and [7]; customarily, we call them $M$-band Daubechies wavelets. An $M$-band $K$-regular Daubechies filters can be described as follows [7]:

$$
\begin{gathered}
H_{M, K}^{\text {Daubechies }}(z)=\left(\frac{z^{M}-1}{M(Z-1)}\right)^{K} Q_{K}(z) \\
\text { where } Q_{K}(z) Q_{K}\left(z^{-1}\right)=\sum_{s=0}^{K-1} a_{M, K}(s)\left(2-z-z^{-1}\right)^{s} \\
a_{M, K}(s) \quad \sum_{s_{1}+s_{2}+\cdots+s_{M-1}=s} \prod_{j=1}^{M-1}\left(\begin{array}{c}
K-1+s_{j} \\
s_{j}
\end{array}\right)\left(2 \sin \frac{j \pi}{M}\right)^{-2 s_{j}} .
\end{gathered}
$$

The another equivalent form appears in [6]. On the other hand, similar to $2 K$-regular Largrange halfband filters, a $2 K$-regular Largrange $M$ th-band interpolating filter is defined as

$$
H_{M, 2 K}^{\text {Lagrange }}(z)=1+\sum_{i=1}^{M-1} \sum_{n} h_{D, M, i}^{(2 K,-K)}(n) z^{-M n-i} .
$$

This filter satisfies the following:

1) Cardinal interpolation;

2) symmetry;

3) support set $\{-M K+1,-M K+2, \ldots, M K-1\}$;

4) $2 K$-regular.

Obviously, it is the unique interpolating filter satisfying these four conditions because its subfilters are completely determined by the linear system of equations in (9). For an $M$-band $K$-regular Daubechies filter, due to orthogonality, $H_{M, K}^{\text {Daubechies }}(z) H_{M, K}^{\text {Daubechies }}\left(z^{-1}\right)$ is an interpolating filter that satisfies 1)-4). From the uniqueness, we have

$$
H_{M, 2 K}^{\text {Lagrange }}(z)=H_{M, K}^{\text {Daubechies }}(z) H_{M, K}^{\text {Daubechies }}\left(z^{-1}\right)
$$

which shows the same relationship holds between the $M$-band Daubechies filters and the Largrange $M$-band interpolating filters. This relationship is useful in fast generation and performance analysis of $M$-band Daubechies wavelets.

\section{Design Method of FIR Regular OSISFs}

At first, we desire that the nonzero coefficients of the scaling filter concentrate near $n=0$, which is a reasonable presuppo- sition to generate a good scaling function. Without loss of generality, assume all subfilters have the same length and support set. In what follows, we discuss two cases: $M$ is an odd integer and $M$ an even integer.

\section{A. $M=2 N+1$ Case}

In this case, there are $2 N+1$ subfilters. According to Theorem 1, for an $M$-band orthogonal symmetric scaling filter with cardinal interpolation, we only need to design its $N$ subfilters $h_{0, i}, i=1,2, \ldots, N$. Let the subfilters $h_{0, i}, i=1,2, \ldots, N$ have the same support set $\{m, m+1, \ldots, m+L-1\}$, where $m=-[L / 2]$, and $[L / 2]$ denotes the integer part of $L / 2$. This assumption can assure that the nonzero coefficients of the scaling filter concentrate near $n=0$. When a scaling filter $h_{0}$ is $K$-regular and $L \geqslant K$, then its subfilters $h_{0, i}$ can be parameterized as

$$
\begin{gathered}
h_{0, i}(n)=\frac{1}{\sqrt{M}} \sum_{k=0}^{L-K} \beta_{i}(k) h_{D, M, i}^{(L-k,-[(L-k) / 2])}(n) \\
i=1,2, \ldots, N .
\end{gathered}
$$

Since the coefficient sum of each Dubuc filter is equal to one and the constraint $\sum h_{0, i}(n)=1 \sqrt{M}$, it is apparent that $\sum \boldsymbol{\beta}_{i}(k)=1$. In (16), the center of the support set of every Dubuc filter is close to $n=0$; thus, their coefficients change in small dynamic range, which is beneficial to numerical calculation. Consequently, designing a $K$-regular OSISF is simplified into selecting the parameter vectors $\boldsymbol{\beta}_{i}$.

Let $\mathbf{Q}_{i, l}$ be a $(L-K+1) \times(L-K+1)$ square matrix whose entries are defined as

$$
\begin{gathered}
q_{i, l}\left(r_{1}, r_{2}\right) \\
=\sum_{k} h_{D, M, i}^{\left(L-r_{1}, m+r_{1}\right)}(k) h_{D, M, i}^{\left(L-r_{2}, m+r_{2}\right)}(k+l) \\
\quad r_{1}, r_{2}=0,1, \ldots, L-K
\end{gathered}
$$

then, $\left|H_{0, i}(\omega)\right|^{2}$ is a cosine polynomial given by

$$
M\left|H_{0, i}(\omega)\right|^{2}=\boldsymbol{\beta}_{i}^{\mathbf{T}} \mathbf{Q}_{i, 0} \beta_{i}+2 \sum_{l=1}^{L-1}\left(\beta_{i}^{\mathbf{T}} \mathbf{Q}_{i, l} \beta_{i}\right) \cos l \omega .
$$

According to Theorem 1, the $K$-regular symmetric interpolating scaling filter $h_{0}$ is orthogonal iff the parameter vectors $\boldsymbol{\beta}_{i}$ are the solutions of the following system of equations:

$$
\begin{aligned}
\sum_{i=1}^{N} \boldsymbol{\beta}_{i}^{\mathbf{T}} \mathbf{Q}_{i, 0} \boldsymbol{\beta}_{i} & =N \\
\sum_{i=1}^{N} \boldsymbol{\beta}_{i}^{\mathbf{T}} \mathbf{Q}_{i, l} \boldsymbol{\beta}_{i} & =0, \quad l=1,2, \ldots, L-1 \\
\sum \boldsymbol{\beta}_{i}(k) & =1 .
\end{aligned}
$$

Obviously, the first equation in (19) is not intrinsic since if the latter $L+N-1$ equations hold, then the first naturally holds. In general, the latter $L+N-1$ equations are mutually independent; thus, in order to guarantee that the system (19) has solutions, 
the number of variables cannot be less than $L+N-1$, that is

$$
L+N-1 \leq N(L-K+1) \quad \text { or } \quad L \geqslant \frac{N K-1}{N-1} .
$$

In the subsequent design, we take $L$ to be the smallest integer satisfying the inequality (20) and denote it as $L(K)$. For example, $L(K)=2 K-1$, when $M=5$.

\section{B. $M=2 N$ Case}

In this case, a $K$-regular OSISF $h_{0}(n)$ has $2 N$ subfilters $h_{0, i}, i=0,1,2, \ldots, 2 N-1$, and the subfilter $h_{0, N}(n)$ must be symmetric, i.e., $h_{0, N}(-(n+1))=h_{0, N}(n)$. Therefore, the length of the support set of the subfilters must be an even number. Let the support set be $\{-L,-L+1, \ldots, L-1\}$; then, the subfilters have the following parametric structure:

$$
\begin{aligned}
h_{0, i}(n)= & \frac{1}{\sqrt{M}} \sum_{k=0}^{2 L-K} \boldsymbol{\beta}_{i}(k) h_{D, M, i}^{(2 L-k,-[(2 L-k) / 2])}(n) \\
& i=1,2, \ldots, N-1 \\
h_{0, N}(n)= & \frac{1}{\sqrt{M}} \sum_{k=0}^{L-[(K+1) / 2]} \boldsymbol{\beta}_{N}(k) h_{D, M, N}^{(2 L-2 k,-(L-k))}(n) .
\end{aligned}
$$

Define the square matrices $\mathbf{Q}_{i, l}$ for $i=1,2, \ldots, N-1$ in a way similar to (18), and define the entries of the square matrices $\mathbf{Q}_{N, l}$ as follows:

$$
\begin{aligned}
& q_{N, l}\left(r_{1}, r_{2}\right) \\
& \quad=\sum_{k} h_{D, M, N}^{\left(2 L-2 r_{1},-\left(L-r_{1}\right)\right)}(k) h_{D, M, N}^{\left(2 L-2 r_{2},-\left(L-r_{2}\right)\right)}(k+l) .
\end{aligned}
$$

A $K$-regular symmetric interpolating scaling filter $h_{0}$ can be designed by solving the following system of quadratic equations:

$$
\begin{aligned}
2 \sum_{i=1}^{N-1} \boldsymbol{\beta}_{i}^{\mathbf{T}} \mathbf{Q}_{i, l} \boldsymbol{\beta}_{i}+\boldsymbol{\beta}_{N}^{\mathbf{T}} \mathbf{Q}_{N, \ell} \boldsymbol{\beta}_{N} & =0, \quad l=1,2, \ldots, 2 L-1 \\
\sum \boldsymbol{\beta}_{i}(k) & =1 .
\end{aligned}
$$

In general, the $2 L+N-1$ equations are mutually independent; in order to make the system of equations be a solution, the number of variables should be greater than or equal to the number of equations, i.e.,

$$
L \geqslant \frac{(N-1) K+\left[\frac{K-1}{2}\right]}{2 N-3} .
$$

Here, $L(K)$ denotes the smallest integer satisfying (23). For example, $L(K)=K+[(K-1) / 2]$, when $M=4$.

\section{Solving the Systems of Equations}

From the above results, solving the systems (19) and (22) becomes the crux of designing FIR regular OSISFs. When the regular order $K$ is smaller, accurate solutions can be computed by hand, and then, the best OSISF is picked out. However, when the regular order $K$ is larger, the numerical methods have to be used. In what follows, when $M=2 N+1$, we define a non-negative objective function

$$
f\left(\boldsymbol{\beta}_{1}, \boldsymbol{\beta}_{2}, \ldots, \boldsymbol{\beta}_{N}\right) \equiv \sum_{l=1}^{L(K)-1}\left(\sum_{i=1}^{N} \beta_{i}^{\mathbf{T}} \mathbf{Q}_{i, l} \boldsymbol{\beta}_{i}\right)^{2}
$$

whereas when $M=2 N$, we define

$$
\begin{aligned}
& f\left(\boldsymbol{\beta}_{1}, \boldsymbol{\beta}_{2}, \ldots, \boldsymbol{\beta}_{N}\right) \\
& \quad \equiv \sum_{l=1}^{2 L(K)-1}\left(2 \sum_{i=1}^{N-1} \boldsymbol{\beta}_{i}^{\mathbf{T}} \mathbf{Q}_{i, l} \boldsymbol{\beta}_{i}+\boldsymbol{\beta}_{N}^{\mathbf{T}} \mathbf{Q}_{N, l} \boldsymbol{\beta}_{N}\right)^{2} .
\end{aligned}
$$

To solve the systems (19) and (22), one can search for the zero points of the object functions subject to the linear constraints $\sum \boldsymbol{\beta}_{\boldsymbol{i}}(k)=1$. Here, the gradient algorithm and Newton's algorithm are used in turn. Starting from a random initial parameter vector, by the gradient algorithm, a solution near a zero point of the objective function is achieved, where the objective function value is less than $10^{-6}$; then, Newton's algorithm is employed in order to quickly achieve a zero point, where the objective function value is less than $10^{-20}$. Due to random initial parameter vectors, many but not all solutions of the systems in (19) and (22) are picked up.

In general, systems (19) and (22) have many solutions, and each solution generates an OSISF. However, the associated scaling functions are very different in smoothness, reflecting the low-bound of the Höllder exponent or Sobolev exponent estimated by (5) and (6). From the obtained solutions, one can choose the best one. Unfortunately, the vanishing moment $K$ is too large, and the above scheme suffers from expensive computational load.

In addition, when $M \geqslant 6$, according to (19) and (22), the number of variables in the system of equations is often more than that of the equations. In this case, the part of the subfilters is fixed in advance, and then, the other subfilters are designed to satisfy the orthogonal condition.

\section{EXAMPLES OF FIR REGULAR OSISFS}

In the following examples, we take the parameter $L=L(K)$, which is the smallest integer satisfying the inequality (20) or (22). First, we consider $K=1,2$ cases; here, the accurate filter coefficients are obtained by hand. For the $K>2$ case, the four-band and five-band OSISFs are designed only with the numerical method in the above section.

\section{A. One-Regular OSISFs}

In the $M=2 N+1$ case, the parameter $L(1)=1$ and the subfilters satisfy $h_{0, i}(n)=(1 / \sqrt{M}) \delta(n+1)$. The associated OSISFs and scaling functions are Haar scaling filters and Haar scaling functions, respectively, and their Sobolev exponents are 0.5 .

In $M=2 N$ case, the parameter $L(1)=2$ and the subfilters satisfy

$$
\begin{aligned}
h_{0, i} & =\frac{1}{\sqrt{M}}\left[\alpha_{i},\left(1-\alpha_{i}\right)\right], \quad i=1,2, \ldots, N-1 \\
h_{0, N} & =\frac{1}{\sqrt{M}}\left[\frac{1}{2}, \frac{1}{2}\right] .
\end{aligned}
$$



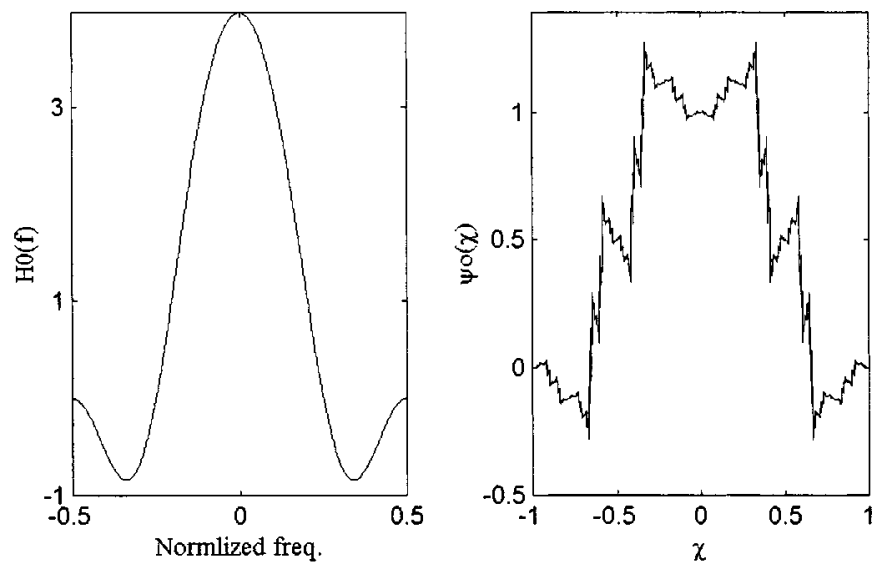

Fig. 1. Four-band one-regular OSISF and scaling function.

The orthogonality is equivalently that the parameters satisfy $\sum_{i=1}^{N-1} 2 \alpha_{i}\left(1-\alpha_{i}\right)+1 / 4=0$. When $M=4$ and $\alpha_{1}=$ $(2 \pm \sqrt{6}) / 4$, the associated OSISFs are, respectively, given by

$$
\begin{aligned}
& h_{0}^{(4,1)}=\frac{1}{8}[2-\sqrt{6}, 2,2+\sqrt{6}, 4,2+\sqrt{6}, 2,2-\sqrt{6}] \\
& h_{0}^{(4,1)}=\frac{1}{8}[2+\sqrt{6}, 2,2-\sqrt{6}, 4,2-\sqrt{6}, 2,2+\sqrt{6}] .
\end{aligned}
$$

The frequency response of the first filter and the associated scaling function are shown in Fig. 1. Clearly, it is known from Fig. 1 that the frequency response has no zero for $\omega \in[-\pi 4, \pi 4]$, the orthonormality of the scaling function is immediately inferred, and the Sobolev exponent of the scaling function is 0.5918 .

When $M=6$, if the parameter vectors $\alpha_{1}, \alpha_{2}$ satisfy the equation

$$
\alpha_{1}\left(1-\alpha_{1}\right)+\alpha_{2}\left(1-\alpha_{2}\right)+1 / 8=0
$$

the corresponding scaling filter is an OSISF. Therefore, six-band one-regular OSISFs can be completely parameterized in the following form:

$$
\begin{aligned}
h_{0}^{(6,1)} & =\left[1-\alpha_{1}, 1-\alpha_{2}, 1 / 2, \alpha_{2}, \alpha_{1}, 1, \ldots, 1-\alpha_{1}\right] \\
\alpha_{1} & =\frac{1}{2}\left[1+\sqrt{\frac{3}{2}+4 \alpha_{2}\left(1-\alpha_{2}\right)}\right] \\
\alpha_{2} & \in\left[\frac{2-\sqrt{10}}{4}, \frac{2+\sqrt{10}}{4}\right]
\end{aligned}
$$

Each $\alpha_{2}$ generates a six-band one-regular OSISF. As two examples, Fig. 2 shows two scaling functions corresponding to $\alpha_{1}=$ $(1 / 4)(2+\sqrt{6}), \alpha_{2}=1$ and $\alpha_{1}=\alpha_{2}=(1 / 2)(1+\sqrt{5 / 4})$, respectively, and their Sobolev exponents are 0.6666 and 0.6281 , respectively.

We see that when $\alpha_{1}=\alpha_{2}$, the scaling function is close to the Haar scaling function in shape. Similarly, with $M=$ $2 N$ increasing, the scaling function with $\alpha_{1}=\alpha_{2}=\cdots=$ $\alpha_{N-1}=(1 / 2)(1+\sqrt{(2 N-1) /(2 N-2)})$ converges to the Haar scaling function or the square wave.
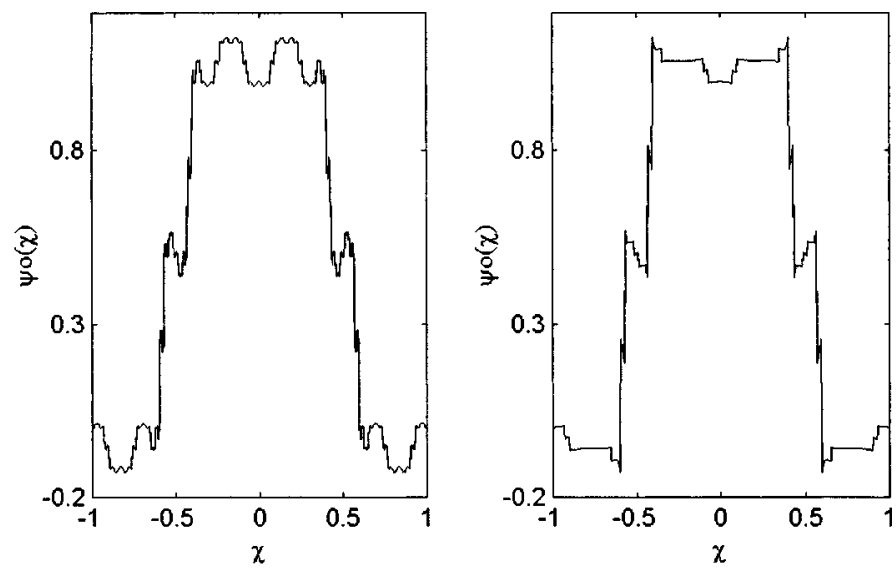

Fig. 2. Two six-band one-regular scaling functions.
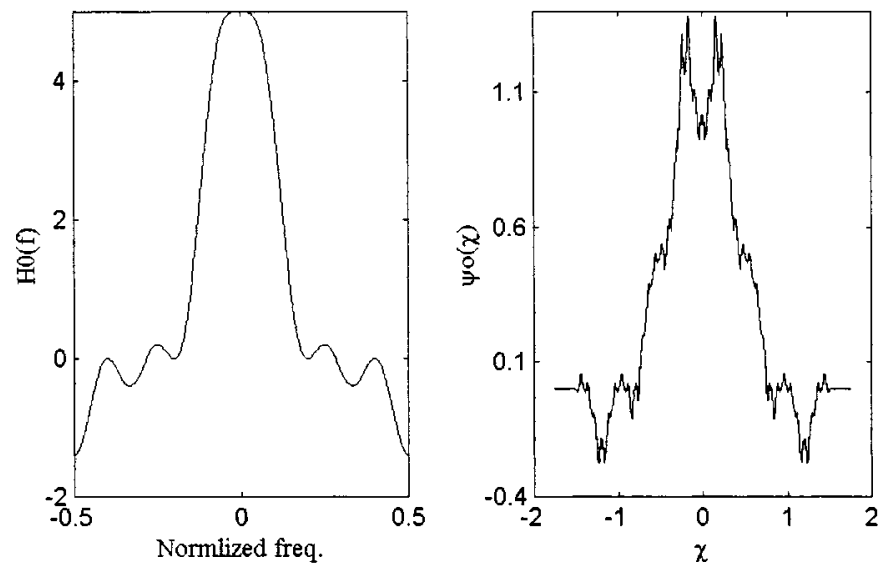

Fig. 3. Five-band two-regular scaling filter and scaling function.

\section{B. Five-Band More Regular OSISFs}

When $K=2$, a set of equations (20) has two solutions, and their corresponding subfilters are, respectively, given by

$$
h_{0,1}=\left[0, \frac{6}{5},-\frac{1}{5}\right], \quad h_{0,2}=\left[\frac{2}{5}, \frac{3}{5}, 0\right]
$$

and

$$
h_{0,1}=\left[\frac{3}{10}, \frac{3}{5}, \frac{1}{10}\right], \quad h_{0,2}=\left[\frac{1}{10}, \frac{6}{5},-\frac{3}{10}\right] .
$$

The first set of subfilters generates a five-band symmetric orthogonal interpolating scaling function with better analytic property, as shown in Fig. 3. Obviously, the frequency response is positive for $\omega \in[-(\pi / 5),(\pi / 5)]$, and the frequency response is factorized as

$$
H_{0}(\omega)=\left(\frac{1+2 \cos (\omega)+2 \cos (2 \omega)}{5}\right)^{2} Q(\omega)
$$

where $Q(\omega)=20 \cos (\omega)-10 \cos (2 \omega)-5$, and $\max _{\omega}|Q(\omega)|=$ 35 . Thus, the scaling function is orthonormal. The low-bound Hölder exponent of the scaling function estimated by (5) is less than 0 . Therefore, a five-band two-regular scaling function is constructed, which has rational filter coefficients, except for the five advantages required by us.

When $K \geqslant 3$, we have to use the numerical algorithms in Section IV-C to solve the system of equations (19). The obtained parameter vectors are given in Tables I and II. The frequency 
TABLE I

PARAMETER VECTORS OF FIVE-BAND THREE AND FOUR REGULAR OSISFS

\begin{tabular}{c|c|c|c|c}
\hline$\beta_{i}$ & $\beta_{1}$ & $\beta_{2}$ & $\beta_{1}$ & $\beta_{2}$ \\
\hline $\mathrm{i}=1$ & 0.34646521185501 & 0.38329427417072 & 0.0184096545624388 & 0.0243416720779421 \\
\hline $\mathrm{i}=2$ & -1.53160523949524 & 0.21531912890462 & -3.63422305219063 & 1.01131152422609 \\
\hline $\mathrm{i}=3$ & 2.18514002764023 & 0.40138659692466 & 3.12594051024182 & 0.922122231301493 \\
\hline $\mathrm{i}=4$ & & & 1.48987288738636 & -0.957775427605534 \\
\hline
\end{tabular}

TABLE II

PARAMETER VECTORS OF FIVE-BAND FIVE AND SIX REgULAR OSISFS

\begin{tabular}{c|c|c|c|c}
\hline$\beta_{i}$ & $\beta_{1}$ & $\beta_{2}$ & $\beta_{1}$ & $\beta_{2}$ \\
\hline $\mathrm{i}=1$ & 0.184487164681813 & 0.429151194420753 & 0.70896344848106 & 0.89213998403580 \\
\hline $\mathrm{i}=2$ & -0.750391152727642 & -0.130237131378472 & 3.75510434143538 & -1.72022968113583 \\
\hline $\mathrm{i}=3$ & 0.282868872549000 & 0.448453032756689 & -3.04254515850165 & -1.04194157094469 \\
\hline $\mathrm{i}=4$ & -0.988709769123080 & -0.354335827261894 & -7.50292650620738 & 2.30073981413547 \\
\hline $\mathrm{i}=5$ & 2.271744884619915 & 0.606968731462919 & 5.11835660517008 & 1.81507145193009 \\
\hline $\mathrm{i}=6$ & & & 1.96304726962251 & -1.24577999802085 \\
\hline
\end{tabular}
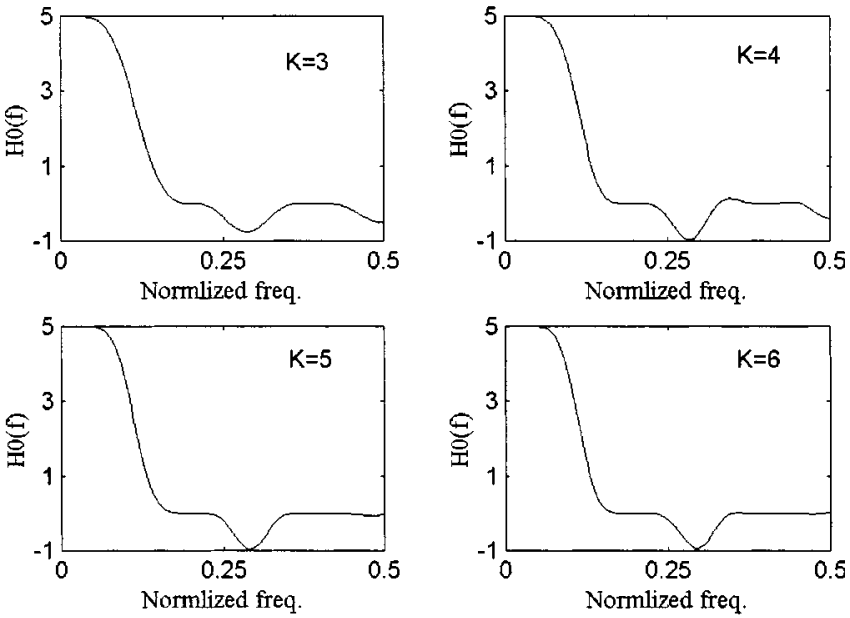

Fig. 4. Frequency responses of five-band three to six regular OSISF's.

responses of three- and six-regular OSISFs are illustrated in Fig. 4, and the three-regular and six-regular scaling functions are shown in Fig. 5, in which the six-regular one is described on its part support set. According to (5) and (6), a low bound of the Höilder exponents and Sobolev of the scaling functions are estimated, as illustrated in Table III.

\section{Four-Band More Regular OSISFs}

When $K=2$, the polyphase filters have the following parametric representations

$$
\begin{aligned}
& h_{0,1}=\left[-\frac{3}{4} \gamma, \frac{1}{4}(1-\beta)+\frac{3}{2} \gamma, \frac{3}{4}(1-\gamma)+\frac{1}{2} \beta,-\frac{1}{4} \beta\right] \\
& h_{0,2}=\left[-\frac{1}{2} \alpha, \frac{1}{2}(1+\alpha), \frac{1}{2}(1+\alpha),-\frac{1}{2} \alpha\right] .
\end{aligned}
$$

According to the orthogonal condition, the parameters $\alpha, \beta$, and $\gamma$ must satisfy the following system of equations:

$$
\begin{aligned}
4 \alpha^{2}+\beta^{2}+9 \gamma^{2}-4 \alpha-\beta-9 \gamma & =0 \\
2 \alpha+\beta+3 \gamma & =5 / 8 \\
2 \alpha^{2}+3 \beta \gamma & =0
\end{aligned}
$$
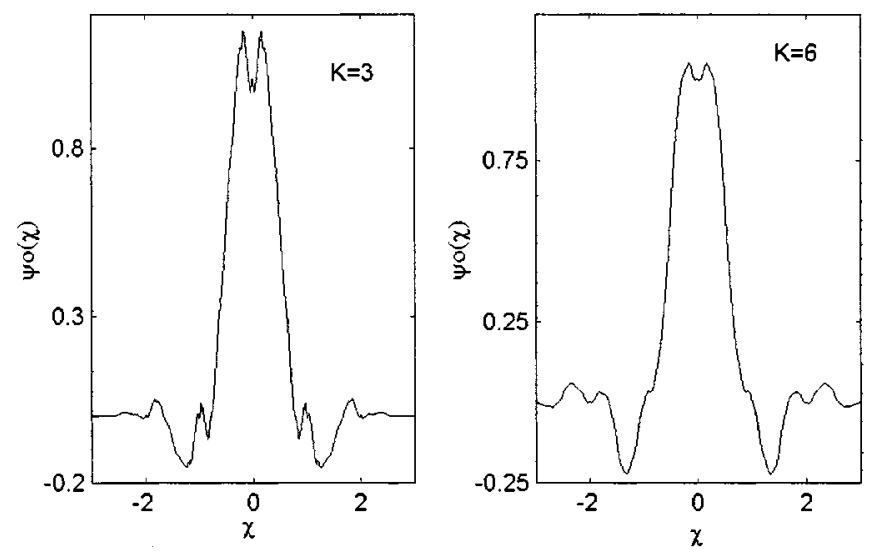

Fig. 5. Three and six regular scaling functions.

TABLE III

HOILDER AND SOBOLEV EXPONENTS OF FIVE-BAND SCALING FunCTIONS

\begin{tabular}{c|c|c|c|c|c}
\hline Regular Order & 2 & 3 & 4 & 5 & 6 \\
\hline Low Bound of $\kappa_{\infty}$ & -0.2091 & 0.4207 & 0.5199 & 0.8992 & 0.9481 \\
\hline$\kappa_{2}$ & 0.7551 & 1.2251 & 1.4319 & 1.7365 & 1.9235 \\
\hline
\end{tabular}

The above system has four solutions where the two solutions corresponding to the better scaling functions are

$$
\begin{array}{ll}
\alpha_{1}=(5+\Theta) / 48 & \alpha_{2}=(5-\Theta) / 48 \\
\beta_{1}=(12 \sqrt{6}-2-\Theta) / 48 & \beta_{2}=(12 \sqrt{6}-2+\Theta) / 48 \\
\gamma_{1}=(22-12 \sqrt{6}-\Theta) / 14 & \gamma_{2}=(22-12 \sqrt{6}+\Theta) / 144
\end{array}
$$

where $\Theta=\sqrt{286-96 \sqrt{6}}$. Interestingly, the second scaling filter is identical to the example in [11], although they are derived from completely different approaches.

When $K>2$, the numerical method is used to obtain the parameter vectors of OSISFs. For $K=3,4,5,6$, the parameter vectors are illustrated in Tables IV and V. From (5) and (6), a low bound of the Höilder exponents and Sobolev exponents of the associated scaling function are shown in Table VI. 
TABLE IV

PARAMETER VeCtors OF FOUR-BAND THREE AND Four REgUlAR OSISFS

\begin{tabular}{c|c|c|c|c}
\hline$\beta_{i}$ & $\beta_{1}$ & $\beta_{2}$ & $\beta_{1}$ & $\beta_{2}$ \\
\hline $\mathrm{i}=1$ & 0.77870241700700 & 0.18480141348693 & 1.18908417038907 & 0.69217117606933 \\
\hline $\mathrm{i}=2$ & -0.38117768511519 & -0.18700532064801 & -0.74652071226967 & -1.35521300497696 \\
\hline $\mathrm{i}=3$ & 1.24108122714595 & 1.00220390716109 & -0.12316264342573 & 2.28589028102117 \\
\hline $\mathrm{i}=4$ & -0.60015497595149 & & 0.48376594155427 & -0.62284845211355 \\
\hline $\mathrm{i}=5$ & 0.77251721566882 & & 1.73458231958560 & \\
\hline $\mathrm{i}=6$ & -0.81096819875510 & & -1.96484515728283 & \\
\hline $\mathrm{i}=7$ & & & 0.42709608144930 & \\
\hline
\end{tabular}

TABLE V

PaRAMETER Vectors of Four-BAND Five AND Six REgUlar OSISFS

\begin{tabular}{c|c|c|c|c}
\hline$\beta_{i}$ & $\beta_{1}$ & $\beta_{2}$ & $\beta_{1}$ & $\beta_{2}$ \\
\hline $\mathrm{i}=1$ & 1.99176848754050 & 1.85731346916586 & 30.13383970066399 & 29.0207975432901 \\
\hline $\mathrm{i}=2$ & -1.77431897659990 & -2.46637311219875 & -27.74488308025162 & -100.163161102052 \\
\hline $\mathrm{i}=3$ & -1.56300698326452 & 4.76135425325253 & -70.31457832704329 & 136.902718643178 \\
\hline $\mathrm{i}=4$ & 1.37887387217341 & -4.59035811399118 & 79.52789453858995 & -85.3517114104807 \\
\hline $\mathrm{i}=5$ & 3.56949879913418 & 1.4380635037715 & 60.97876822620857 & 21.6996982892195 \\
\hline $\mathrm{i}=6$ & -3.90360135970715 & & -92.83485847099898 & -1.10834196315325 \\
\hline $\mathrm{i}=7$ & -0.29637806383649 & & -12.24353030031760 & \\
\hline $\mathrm{i}=8$ & 2.03317655748858 & & 43.73679700667947 & \\
\hline $\mathrm{i}=9$ & 0.40406112103364 & & -4.47357593876446 & \\
\hline $\mathrm{i}=10$ & -0.84007345396225 & & -6.53365200945357 & \\
\hline $\mathrm{i}=11$ & & & 0.76777865468667 & \\
\hline
\end{tabular}

TABLE VI

HoIldER AND SOBOlEv EXPONENTS OF Four-BAND SCALING Functions

\begin{tabular}{c|c|c|c|c|c|c}
\hline Low Bound of $\kappa_{\infty}$ & 0.0762 & 0.0761 & 0.3556 & 0.5303 & 0.7083 & 0.8504 \\
\hline$\kappa_{2}$ & 0.9678 & 0.8904 & 1.1828 & 1.3451 & 1.7414 & 1.8534 \\
\hline
\end{tabular}

In numerical computation, the final parameter vectors correspond to the value of the objective function less than $10^{-20}$, which is enough for most of applications. When $M \geqslant 6$, the similar algorithms can be employed to design OSISFs.

\section{CONCLUSION}

This paper proposed a novel approach to designing the $M$-band $(M \geqslant 4)$ FIR OSISFs and compactly supported orthogonal symmetric interpolating scaling functions. Using $M$-band Dubuc filters, we obtained the parametric representations of $M$-band $K$-regular symmetric cardinal interpolating scaling filters. The orthogonality imposes the quadric constraints in the parameter vectors. Solving a set of quadric equations by hand or the numerical algorithms, two family of five-band and four-band compactly supported orthogonal symmetric cardinal interpolating scaling functions are designed. These are an absolutely necessary preparation to designing FIR orthogonal linear-phase interpolating filterbanks and wavelets. Such filterbanks and wavelets are required in most applications such as image processing and singularity detection. From these scaling functions, one can design compactly supported linear-phase orthogonal interpolating wavelets. There exist several approaches to construct a linear-phase paraunitary filterbank or wavelets from a linear-phase scaling filter [4]-[6], but these approaches have some limitations on the initial scaling filter or $M$; therefore, it is nontrivial to design a linear-phase interpolating filter bank or wavelet from an OSISF. The design approach proposed in [11] is an important reference in designing linear-phase interpolating filterbanks and wavelets, which is based on the recursive structure on the band number $M$.

\section{ACKNOWLEDGMENT}

The authors gratefully thank the reviewers for their many useful suggestions that improved the paper significantly.

\section{REFERENCES}

[1] I. Daubechies, "Orthonormal bases of compactly supported wavelets," Commun. Pure Appl. Math., vol. 41, pp. 909-996, 1988.

[2] C. Chui and J. A. Lian, "Construction of compactly supported symmetric and antisymmetric orthonormal wavelets with scale=3," Appl. Comput. Harmon. Anal., vol. 2, pp. 21-51, 1995.

[3] E. Belogay and Y. Wang, "Compactly supported orthogonal symmetric scaling functions," Appl. Comput. Harmon. Anal., vol. 7, pp. 137-150, 1999.

[4] A. K. Soman, P. P. Vaidyanathan, and T. Q. Nguong, "Linear phase paraunitary filter banks: Theory, factorizations and designs," IEEE Trans. Signal Processing, vol. 41, pp. 3480-3496, Dec. 1993.

[5] O. Alkin and H. Caglar, "Design of efficient $M$-band coder with linearphase and perfect-reconstruction properties," IEEE Trans. Signal Processing, vol. 45, pp. 1579-1590, July 1995.

[6] P. Steffen, P. N. Heller, R. A. Gopinath, and C. S. Burrus, "Theory of regular $M$-band wavelet bases," IEEE Trans. Signal Processing, vol. 41, pp. 3497-3510, Dec. 1993

[7] N. Bi, X. Dai, and Q. Sun, "Construction of compactly supported $M$-band wavelets," Appl. Comput. Harmon. Anal., vol. 6, pp. 113-131, 1999.

[8] J.-K. Zhang and Z. Bao, "Three-band compactly supported orthogonal interpolating scaling function," Electron. Lett., vol. 34, no. 5, pp. 451-452, 1998. 
[9] L. Monzon and G. Beylkin, "Compactly supported wavelets bases on almost interpolating and nearly linear phase filters," Appl. Comput. Harmon. Anal., vol. 7, pp. 184-210, 1999.

[10] I. W. Selesnick, "Interpolating multiwavelet bases and the sampling theorem," IEEE Trans. Signal Processing, vol. 47, pp. 1615-1621, June 1999.

[11] H. Ji and Z. Shen, "Compactly supported (bi)orthogonal wavelets generated by interpolatory refinable functions," Adv. Comput. Math., vol. 11 , pp. 81-104, 1999

[12] X.-G. Xia and Z. Zheng, "On sampling theorem, wavelets, and wavelet transforms," IEEE Trans. Signal Processing, vol. 41, pp. 3524-3535, Feb. 1993

[13] G. G. Walter, "A sampling theorem for wavelets subspaces," IEEE Trans. Inform. Theory, vol. 38, pp. 881-884, Apr. 1992.

[14] A. Aldroubi and M. Unser, "Families of wavelets transforms in connection with Shannon's sampling theory and the Gabor transform," in Wavelets: A Tutorial in Theory and Applications, C. K. Chui, Ed. New York: Academic, 1992, pp. 509-528.

[15] O. Rioul and P. Duhamel, "Fast algorithms for discrete and continuous wavelet transforms," IEEE Trans. Inform. Theory, vol. 38, pp. 569-586, Apr. 1992

[16] D. L. Donoho, "Interpolating wavelet transform," Tech. Rep., Dept. Statistics, Stanford Univ., Stanford, CA, Oct. 1992.

[17] W. Sweldens, "The lifting scheme: A construction of second generation wavelets," SIAM J. Math. Anal., vol. 29, no. 2, pp. 511-546, 1997.

[18] R. Ansari, C. Guillemot, and J. F. Kaiser, "Wavelet construction using Largrange halfband filters," IEEE Trans. Circuits Syst., vol. 38, pp. 1116-1118, June 1991.

[19] S. Dubuc, "Interpolation through an iterative scheme," J. Math. Anal. Appl., vol. 144, pp. 185-204, 1986.

[20] N. J. Fliege, Multirate Digital Signal Processing: Wiley, 1994, pp. 188-203.

[21] P.-L. Shui and Z. Bao, "Construction of nearly orthogonal interpolating wavelets," Signal Process., vol. 70, no. 3, pp. 289-300, 1999.

[22] I. Daubechies and J. C. Lagarias, "Two-scale different equations-I: Existence and global regularity of solutions," SIAM J. Math. Anal., vol. 22, no. 5 , pp. $1388-1410,1991$.

[23] — "Two-scale different equations-II: Local regularity, infinite products of matrices and fractals," SIAM J. Math. Anal., vol. 23, no. 4, pp. 1031-1079, 1992.

[24] M. Ali and A. H. Tewfik, "Multiscale different equation signal model: Part I-Theory," IEEE Trans. Signal Processing, vol. 43, pp. 2332-2345, Oct. 1995

[25] M. J. Shensa, "The discrete wavelet transform: Wedding the a trous and Mallat algorithms," IEEE Trans. Signal Processing, vol. 40, pp. 2464-2482, Oct. 1992

[26] I. Daubechies, "Ten lectures on wavelets," in CBMS Conference Series in Applied Mathematics. Philadelphia, PA: SIAM, 1992, vol. 61.

[27] S. D. Riemenschneider and Z. Shen, "Multidimensional interpolatory subdivision schemes," SIAM J. Numer. Anal., vol. 34, no. 6, pp. 2357-2381, 1997.

[28] K. S. Lau, M. F. Ma, and J. Wang, "On some sharp regularity estimations of $\mathrm{L}^{2}$-scaling functions," SIAM J. Math. Anal., vol. 27, pp. 835-864, 1996.

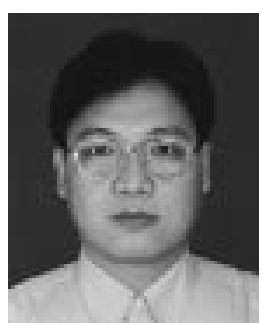

Peng-Lang Shui received the M.S. degree in mathematics from Nanjing University, Nanjing, China, and the $\mathrm{Ph} . \mathrm{D}$. degree in signal and information processing from Xidian University, Xi'an, China, in 1992 and 1999, respectively.

$\mathrm{He}$ is now an Associate Professor with the Department of Applied Mathematics and the Key Laboratory for Radar Signal Processing, Xidian University. His research interests include digital signal processing, wavelets and multirate filterbanks, data compression, fractal theory, and ultra wideband radar signal processing. He has published over 20 papers.

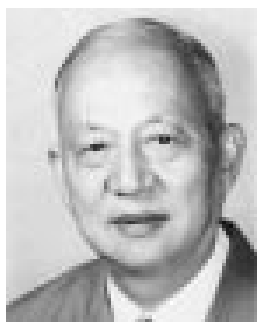

Zheng Bao (M'80-SM'90) received the B.S. degree in radar engineering from Xidian University, Xi'an, China.

$\mathrm{He}$ is now a Professor at Xidian University. He has been working in a number of areas, including radar systems, signal processing, neural networks, and automatic target recognition. He has published more than 200 journal papers and is the author or co-author of ten books. His current research interests include radar signal processing, array signal processing, and SAR/ISAR imaging.

Prof. Bao is a member of the Chinese Academy of Sciences.

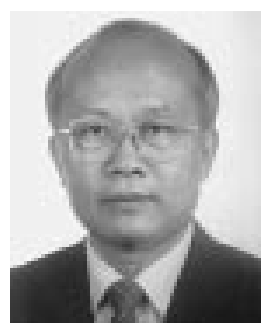

Xian-Da Zhang (SM'93) was born in Jiangxi, China, on May 10, 1946. He graduated from Xidian University, Xi' an, China, in 1970. He received the M.S. degree in instrument engineering from Harbin Institute of Technology, Harbin, China, in 1992 and the Ph.D. degree in electrical engineering from Tohoku University, Sendai, Japan, in 1987.

From August 1990 to August 1991, he was a postdoctoral researcher with the Department of Electrical and Computer Engineering, University of California, San Diego. Since 1992, he has been a Professor with the Department of Automation, Tsinghua University, Beijing, China, and is currently with the Key Laboratory for Radar Signal Processing, Xidian University, as a specially appointed professor awarded by the Minister of Education of China and Cheung Kong Scholars Programme. His current research interests are signal processing with applications in radar and communications and intelligent signal processing. He has published over 80 papers and is the author or co-author of three textbooks and two books. He holds one patent. 